\title{
Projecting the Flow Variables for Hub Location Problems
}

\author{
Martine Labbé \\ Université Libre de Bruxelles, Département d'Informatique, Boulevard du Triomphe CP 210/01, \\ 1050 Bruxelles, Belgium
}

\author{
Hande Yaman \\ Bilkent University, Department of Industrial Engineering, 06800 Bilkent, Ankara, Turkey
}

\begin{abstract}
We consider two formulations for the uncapacitated hub location problem with single assignment (UHL), which use multicommodity flow variables. We project out the flow variables and determine some extreme rays of the projection cones. Then we investigate whether the corresponding inequalities define facets of the UHL polyhedron. We also present two families of facet defining inequalities that dominate some projection inequalities. Finally, we derive a family of valid inequalities that generalizes the facet defining inequalities and that can be separated in polynomial time. ( $) 2004$ Wiley Periodicals, Inc. NETWORKS, Vol. 44(2), 84-93 2004
\end{abstract}

Keywords: hub location; projection; dicut inequalities; polyhedral analysis

\section{INTRODUCTION}

In this article, we consider the Uncapacitated Hub Location Problem with Single Assignment (UHL). Let I denote the set of terminal nodes with $|I|=n$ and $K$ the set of commodities. For commodity $k \in K, o(k)$ is the origin, $d(k)$ is the destination and $t^{k}$ is the amount of traffic where $t^{k}=t_{o(k) d(k)}$. Origins and destinations of commodities are terminal nodes, and any distinct pair of terminal nodes defines a commodity.

Each terminal either receives a $h u b$ or is connected to another node that receives a hub. If node $i \in I$ is connected to such a node $j \in \Lambda\{i\}$, then the traffic on the link between nodes $i$ and $j$ is the traffic adjacent at node $i$, that is, the total traffic of commodities with node $i$ as origin or destination. The cost of routing this traffic on the link between node $i$ and node $j$ is denoted by $F_{i j}$. Any node $i$ that becomes a hub

Received June 2003; accepted January 2004

Correspondence to: H. Yaman; e-mail: hyaman @ bilkent.edu.tr

Contract grant sponsor: France Telecom R\&D; contract grant number: 99 1B 774

DOI 10.1002/net.20019

Published online

in Wiley InterScience (www.interscience.

wiley.com)

(C) 2004 Wiley Periodicals, Inc. is assigned to itself. The cost of installing a hub at node $i$ is denoted by $F_{i i}$.

Let $A=\{(j, l): j \in I, l \in I, j \neq l\}$ and $R_{j l}$ denote the cost of routing a traffic unit on $\operatorname{arc}(j, l)$ if it becomes a backbone arc, that is, if both nodes $j$ and $l$ receive hubs. We assume that the cost vector $R$ satisfies the triangle inequality and $R_{j l} \geq 0$ for all $(j, l) \in A$. In addition, we assume that all data are rational.

If two nodes $i$ and $m$ are assigned to the same hub, say $j$, the traffic from node $i$ to node $m$ follows the path $i \rightarrow$ $j \rightarrow m$. However, if node $i$ is assigned to node $j$ and node $m$ is assigned to node $l$, then the traffic from node $i$ to node $m$ follows the path $i \rightarrow j \rightarrow l \rightarrow m$. Therefore, the total traffic on $\operatorname{arc}(j, l)$ is the sum of the traffic of commodities whose origins are assigned to node $j$ and whose destinations are assigned to node $l$. In Figure 1, we see a network with 10 nodes where nodes $1,2,3$, and 4 are hub nodes. The traffic from node 9 to node 5 follows the path $9 \rightarrow 1 \rightarrow 4$ $\rightarrow 5$, because node 9 is assigned to node 1 and node 5 is assigned to node 4 . The traffic from node 8 to node 7 goes through the path $8 \rightarrow 2 \rightarrow 7$, as both nodes 8 and 7 are assigned to node 2 . Finally, the traffic from node 3 to node 10 goes from node 3 to node 1 and then to node 10 , because node 3 is a hub and so is assigned to itself and node 10 is assigned to node 1 .

The aim of UHL is to choose the hub locations and assign the terminal nodes to hubs to minimize the total cost of location and routing. It has applications in transportation and telecommunication. The UHL is an NP-hard problem (see Yaman [10] for the proof for the special case where $R_{j l}$ $=0$ for all $(j, l) \in A)$. For a recent survey on applications and solution methods, see Campbell et al. [3].

Let $x_{i j}$ be 1 if node $i \in I$ is assigned to node $j \in I$ and 0 otherwise. If node $i$ receives a hub then $x_{i i}$ is 1 and node $i$ is assigned to itself. Further, define $z_{j l}$ to be the total traffic on the $\operatorname{arc}(j, l) \in A$. We can formulate UHL as follows:

$$
\min \sum_{i \in I} \sum_{j \in I} F_{i j} x_{i j}+\sum_{(j, l) \in A} R_{j l} z_{j l}
$$




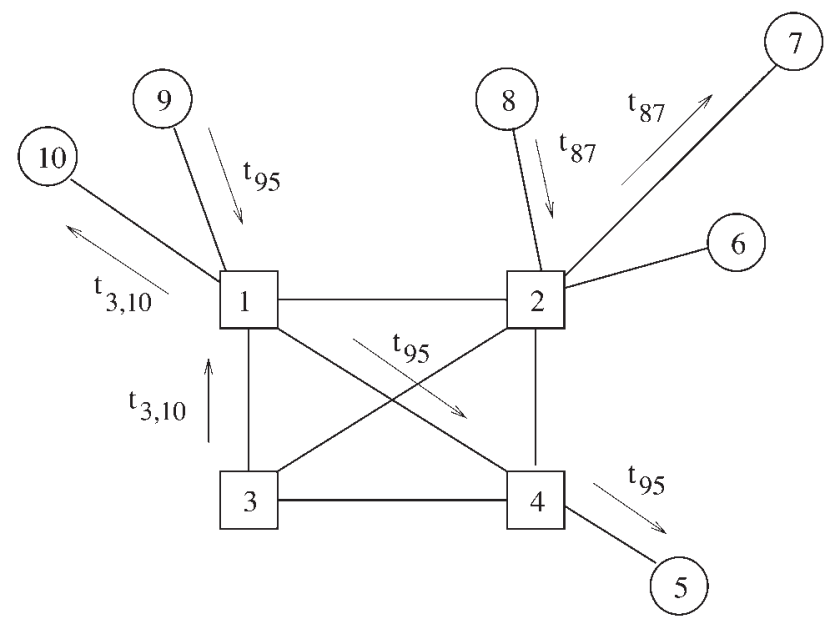

FIG. 1. Routing in a hub network.

$$
\begin{gathered}
\text { s.t. } \sum_{j \in I} x_{i j}=1 \quad \forall i \in I \\
x_{i j} \leq x_{j j} \quad \forall i, j \in I, i \neq j \\
z_{j l} \geq \sum_{k \in K} t^{k} x_{o(k) j} x_{d(k) l} \quad \forall(j, l) \in A \\
x_{i j} \in\{0,1\} \quad \forall i, j \in I \\
z_{j l} \geq 0 \quad \forall(j, l) \in A
\end{gathered}
$$

Constraints (1), (2), and (4) imply that each node either receives a hub or is assigned to exactly one other node which receives a hub. For $(i, j) \in A$ such that $R_{j l}>0$, constraints (3) compute the value of traffic in terms of the assignment variables.

In this article, we present two ways of linearizing constraints (3). These linearizations use the flow variables $X_{j l}^{k}$ $=x_{o(k) j} x_{d(k) l}$ for $k \in K$ and $j \in I, l \in I$ and they have the same linear programming bound (see Yaman [10]). The number of flow variables is $O\left(n^{4}\right)$. So these linearizations are huge in size and not very useful in practice. We discuss how to project out these flow variables to obtain a formulation of a smaller size. Then we investigate the domination among the projection inequalities. Finally, we prove that some of these inequalities are facet defining for the hub location polyhedron. We also introduce two other families of facet-defining inequalities that dominate some of the projection inequalities. Then we give a family of valid inequalities which generalizes the facet defining inequalities.

This article is organized as follows: In Section 2, we present the multicommodity formulation. We project out the flow variables in two different ways and compare the projection inequalities. In Section 3 , we project out the flow variables in the hub location formulation and characterize the extreme rays of the projection cone for a single com- modity. Then we present some families of extreme rays for the multicommodity case. In Section 4, we summarize the previous polyhedral results for the UHL and present new facet-defining inequalities.

\section{MULTICOMMODITY FLOW LINEARIZATION AND ITS PROJECTIONS}

As the routing cost $R$ satisfies the triangle inequality, we can formulate UHL using multicommodity flows. To obtain the multicommodity flow formulation, we replace constraints (3) with the following set of inequalities:

$$
\sum_{l \in \Lambda\{j\}} X_{l j}^{k}-\sum_{l \in \Lambda\{j\}} X_{j l}^{k} \leq x_{d(k) j}-x_{o(k) j} \quad \forall j \in I, k \in K
$$

$$
\begin{aligned}
\sum_{k \in K} t^{k} X_{j l}^{k} \leq z_{j l} & \forall(j, l) \in A \\
X_{j l}^{k} \geq 0 \quad & \forall(j, l) \in A, k \in K
\end{aligned}
$$

Notice that the flow balance equations are replaced by the equivalent inequality forms (6) (see Mirchandani [6]). These constraints state that if the origin of commodity $k$ is assigned to hub $j$ but not the destination, there is a net flow of one unit that goes out of hub $j$. On the contrary, if the destination is assigned to $j$ but not the origin, there is a net flow of one unit that comes into node $j$. If both the origin and destination are assigned to $j$ or neither one is assigned to $j$, the flow on the arcs incoming to node $j$ is equal to the flow on the arcs outgoing from node $j$ concerning this commodity. As the routing cost satisfies the triangle inequality, these constraints guarantee that the traffic of a commodity uses the direct link between the hubs of its origin and destination if its origin and destination are assigned to different hubs.

Constraints (7) imply that the traffic on arc $(j, l)$ should be at least the sum of the traffic of commodities whose origins are assigned to node $j$ and whose destinations are assigned to node $l$. We sometimes refer to the traffic on the backbone links as capacity to be able to follow the terminology of flows and cuts.

To our knowledge, there are two ways of projecting out the flow variables in this system. The first method used by Mirchandani [6] is a direct projection. This method leads to inequalities known as the metric inequalities (see Iri [4] and Onaga and Kakusho [7]). Mirchandani [6] studies the extreme rays of the resulting cone for the single commodity and multicommodity cases. For the single commodity case the projection inequalities are the well-known cut inequalities. However, for the multicommodity case, we do not know any characterization of all the extreme rays of the resulting cone.

The second method used by Rardin and Wolsey [8] is to replace the flow constraints by the corresponding cut con- 
straints and do the projection afterwards. The projection inequalities are called dicut inequalities.

Here, we do the projection using both methods and compare the results. The comparison gives us a necessary condition for the dicut inequalities not to be dominated.

\subsection{Projection 1: Direct Method}

First, we follow the method used by Mirchandani [6]. If we associate dual variables $\alpha_{j}^{k}$ to constraints (6) and $\beta_{j l}$ to constraints (7), by Farkas' Lemma, we have the following result: given a solution $x$ and $z$, there exists a vector $X$ satisfying (6)-(8) if and only if

$$
\sum_{(j, l) \in A} z_{j l} \beta_{j l} \geq \sum_{k \in K} \sum_{j \in I} t^{k}\left(x_{o(k) j}-x_{d(k) j}\right) \alpha_{j}^{k}
$$

for all $(\alpha, \beta) \geq 0$ such that

$$
\beta_{j l} \geq \alpha_{j}^{k}-\alpha_{l}^{k} \quad \forall k \in K,(j, l) \in A
$$

For $a \in \mathbb{R}$, let $(a)^{+}=\max \{0, a\}$. As $z_{j l} \geq 0$ for all $(j$, $l) \in A$ and all the data are rational, for a given $(x, z)$ there exists a vector $X$ that satisfies (6)-(8) if and only if

$$
\sum_{(j, l) \in A} z_{j l} \max \left(\alpha_{k \in K}^{k}-\alpha_{l}^{k}\right)^{+} \geq \sum_{k \in K} \sum_{j \in I} t^{k}\left(x_{o(k) j}-x_{d(k) j}\right) \alpha_{j}^{k}
$$

for all integer $\alpha_{j}^{k} \geq 0$.

\subsection{Projection 2: Indirect Method}

We now do the projection as in Rardin and Wolsey [8]. Let $u_{a}^{k}$ denote the capacity of arc $a$ used for commodity $k$. For $x$, which satisfies (1), (2), and (4), there exists a feasible flow for commodity $k$ if and only if

$$
\sum_{a \in \delta(S)} u_{a}^{k} \geq t^{k} \sum_{j \in S}\left(x_{o(k) j}-x_{d(k) j}\right) \quad \forall S \subseteq I
$$

where $\delta(S)=\{(j, l) \in A: j \in S, l \in \Lambda S\}$ is the cut induced by cut set $S$. So the inequalities (6)-(8) can be replaced by the following:

$$
\begin{gathered}
\sum_{a \in \delta(S)} u_{a}^{k} \geq t^{k} \sum_{j \in S}\left(x_{o(k) j}-x_{d(k) j}\right) \quad \forall S \subseteq I, k \in K \\
\sum_{k \in K} u_{a}^{k}=z_{a} \quad \forall a \in A \\
u_{a}^{k} \geq 0 \quad \forall a \in A, k \in K
\end{gathered}
$$

If we associate dual variables $\gamma_{S}^{k} \geq 0$ to inequalities (12) and $\sigma_{a}$ to equations (13) we get that, given $(x, z)$ which satisfies (1), (2), (4), and (5) there exists a vector $X$ satisfying (6)-(8) if and only if

$$
\sum_{a \in A} z_{a} \sigma_{a} \geq \sum_{S \subseteq I} \sum_{k \in K} t^{k} \sum_{j \in S}\left(x_{o(k) j}-x_{d(k) j}\right) \gamma_{S}^{k}
$$

for all $(\gamma, \sigma)$ such that

$$
\begin{gathered}
\sigma_{a} \geq \sum_{S: a \in \delta(S)} \gamma_{S}^{k} \quad \forall k \in K, a \in A \\
\gamma_{S}^{k} \geq 0 \quad \forall k \in K, S \subseteq I
\end{gathered}
$$

This condition is equivalent to

$$
\sum_{a \in A} z_{a} \max _{k \in K}\left\{\sum_{S: a \in \delta(S)} \gamma_{S}^{k}\right\} \geq \sum_{S \subseteq I} \sum_{k \in K} t^{k} \sum_{j \in S}\left(x_{o(k) j}-x_{d(k) j}\right) \gamma_{S}^{k}
$$

for all integer $\gamma \geq 0$ as all data are rational and $z_{a} \geq 0$ for all $a \in A$.

The $\gamma_{S}^{k}$ s are interpreted in Rardin and Wolsey [8] as follows: if we let $\Gamma^{k}$ denote a collection of cut sets for commodity $k$, we can interpret $\gamma_{S}^{k}$ as the number of times cut set $S$ is repeated in the collection $\Gamma^{k}$.

\subsection{Comparison of Projections 1 and 2}

Proposition 1. For a given $\gamma$, define $\Gamma^{k}$ to be the collection of cut sets for $k \in K$, that is, $S$ is repeated $\gamma_{S}^{k}$ times in $\Gamma^{k}$. Then for a given $(x, z)$ which satisfies (1), (2), (4), and (5), there exists a vector $X$ that satisfies (6)-(8) if and only if (16) is satisfied for all integer $\gamma \geq 0$ such that we can rename the cut sets in $\Gamma^{k}$ as $S_{1}^{k}, \ldots, S_{n_{k}-1}^{k}, S_{n_{k}}^{k}$ where $n_{k}$ $=\left|\Gamma^{k}\right|$ in such a way that $S_{n_{k}}^{k} \subseteq S_{n_{k}-1}^{k} \subseteq \cdots \subseteq S_{1}^{k}$ for all $k$ $\in K$.

Proof. Consider inequality (15) for $(\gamma, \sigma) \geq 0$ such that $\gamma$ is integer and $\sigma_{a}=\max _{k \in K}\left\{\sum_{S \subseteq I: a \in \delta(S)} \gamma_{S}^{k}\right\}$ for all $a \in A$. Define $\alpha_{j}^{k}=\sum_{S \subseteq I: j \in S} \gamma_{S}^{k}$. Then $\alpha_{j}^{k}$ is the number of times node $j$ is repeated in the cut sets in collection $\Gamma^{k}$. The right hand side of inequality (15) is equal to

$$
\begin{aligned}
\sum_{S \subseteq I} \sum_{k \in K} \sum_{j \in S} t^{k}\left(x_{o(k) j}-x_{d(k) j}\right) & \gamma_{S}^{k} \\
& =\sum_{k \in K} \sum_{j \in I} \sum_{S \subseteq I: j \in S} t^{k}\left(x_{o(k) j}-x_{d(k) j}\right) \gamma_{S}^{k} \\
& =\sum_{k \in K} \sum_{j \in I} t^{k}\left(x_{o(k) j}-x_{d(k) j}\right) \alpha_{j}^{k}
\end{aligned}
$$

which is equal to the right-hand side of inequality (9) for this $\alpha$. 
Now we compare the left hand sides. For $(j, l) \in A$ and $k \in K$,

$$
\begin{aligned}
\left(\alpha_{j}^{k}-\alpha_{l}^{k}\right)^{+}= & \left(\sum_{S \subseteq I: j \in S} \gamma_{S}^{k}-\sum_{S \subseteq I: l \in S} \gamma_{S}^{k}\right)^{+} \\
& \leq\left(\sum_{S \subseteq I: j \in S} \gamma_{S}^{k}-\sum_{S \in \Gamma^{k}: l \in S, j \in S} \gamma_{S}^{k}\right)^{+}=\sum_{S \subseteq I:(j, l) \in \delta(S)} \gamma_{S}^{k}
\end{aligned}
$$

Let $k^{\prime}$ be such that $\max _{k \in K}\left(\alpha_{j}^{k}-\alpha_{l}^{k}\right)^{+}=\left(\alpha_{j}^{k^{\prime}}-\alpha_{l}^{k^{\prime}}\right)^{+}$. It follows that

$$
\begin{aligned}
\sigma_{j l}=\max _{k \in K}\left\{\sum_{S \subseteq I:(j, l) \in \delta(S)} \gamma_{S}^{k}\right\} \geq \sum_{S \subseteq I:(j, l) \in \delta(S)} \gamma_{S}^{k^{\prime}} \\
\quad \geq \max _{k \in K}\left(\alpha_{j}^{k}-\alpha_{l}^{k}\right)^{+}=\beta_{j l}
\end{aligned}
$$

Hence, the left-hand side of inequality (15) is greater than or equal to the left-hand side of inequality (9). As the righthand sides are equal, inequality (9) dominates inequality (15).

Next, we show how we can construct a pair $(\gamma, \sigma)$ that gives the same inequality as a given $(\alpha, \beta)$ pair. For each commodity $k$, let $\alpha^{k}=\max _{j \in I} \alpha_{j}^{k}$. Define $\Gamma^{k}$ to be the collection of sets $S_{i}^{k}$ for $i=1,2, \ldots, \alpha^{k}$ where $S_{i}^{k}=\{j$ $\left.\in I: \alpha_{j}^{k} \geq i\right\}$ so that we have $S_{\alpha^{k}}^{k} \subseteq S_{\alpha^{k}-1}^{k} \subseteq \cdots \subseteq S_{1}^{k}$ for all $k \in K$. Define also $\gamma_{S_{i}^{k}}^{k}$ to be the number of times $S_{i}^{k}$ is repeated in $\Gamma^{k}$ for all $i=1,2, \ldots, \alpha^{k}$ and $k \in K$. For $S$ $\notin \Gamma^{k}$, set $\gamma_{S}^{k}=0$. Then $\alpha_{j}^{k}=\sum_{S \subseteq I: j \in S} \gamma_{S}^{k}$. Moreover, if $\alpha_{j}^{k}>\alpha_{l}^{k}$, then whenever $l \in S$, we have $j \in S$. Therefore,

$$
\begin{aligned}
\alpha_{j}^{k}-\alpha_{l}^{k}=\sum_{S \subseteq I: j \in S} \gamma_{S}^{k}-\sum_{S \subseteq I: l \in S} \gamma_{S}^{k} \\
=\sum_{S \subseteq I: j \in S} \gamma_{S}^{k}-\sum_{S \in \Gamma^{k}: l \in S, j \in S} \gamma_{S}^{k}=\sum_{S \subseteq I:(j, l) \in \delta(S)} \gamma_{S}^{k}
\end{aligned}
$$

and if $\alpha_{j}^{k} \leq \alpha_{l}^{k}$, then there is no $S_{i}^{k} \subseteq I$ such that $(j, l)$ $\in \delta\left(S_{i}^{k}\right)$. So

$$
\sum_{S \subseteq I:(j, l) \in \delta(S)} \gamma_{S}^{k}=0=\left(\alpha_{j}^{k}-\alpha_{l}^{k}\right)^{+}
$$

This proves that

$$
\left(\alpha_{j}^{k}-\alpha_{l}^{k}\right)^{+}=\sum_{S \subseteq I:(j, l) \in \delta(S)} \gamma_{S}^{k}
$$

for all $(j, l) \in A$ and $k \in K$.

Hence, for inequalities (16), it is enough to consider $\Gamma$ where we can sort the cut sets in each $\Gamma^{k}$ such that $S_{n_{k}}^{k} \subseteq$ $S_{n_{k}-1}^{k} \subseteq \cdots \subseteq S_{1}^{k}$ where $n_{k}$ is the number of cut sets in $\Gamma^{\bar{k}}$.

This result implies that the inequalities (16) that do not satisfy the condition of Proposition 1 are dominated by inequalities (11). So in a solution procedure based on cuts, one does not need to separate these dominated inequalities.

\section{HUB LOCATION LINEARIZATION AND ITS PROJECTION}

To obtain the hub location linearization, we replace constraints (3) by

$$
\begin{gathered}
\sum_{l \in I} X_{j l}^{k} \geq x_{o(k) j} \quad \forall j \in I, k \in K \\
-\sum_{j \in I} X_{j l}^{k} \geq-x_{d(k) l} \quad \forall l \in I, k \in K \\
-\sum_{k \in K} t^{k} X_{j l}^{k} \geq-z_{j l} \quad \forall(j, l) \in A \\
X_{j l}^{k} \geq 0 \quad \forall(j, l) \in A, k \in K
\end{gathered}
$$

This formulation is given in Skorin-Kapov et al. [9]. Note that we replaced constraints (17) and (18), which are originally equalities by their equivalent inequality forms. This formulation is different from the multicommodity formulation given in the previous section as it explicitly imposes that every commodity travels from the hub of its origin to the hub of its destination directly. If the origin and destination of a commodity $k$ are assigned to the same hub, say $j$, then the variable $X_{j j}^{k}$ takes value 1 (these variables did not exist in the multicommodity formulation).

Proposition 2. Given $(x, z)$, there exists $X$ that satisfies (17)-(20) if and only if

$$
\sum_{a \in A} z_{a} \beta_{a} \geq \sum_{k \in K} t^{k} \sum_{j \in I}\left(x_{o(k) j} \alpha_{j}^{k}-x_{d(k) j} \sigma_{j}^{k}\right)
$$

for all $(\alpha, \sigma, \beta) \geq 0$ such that

$$
\begin{gathered}
\beta_{j l} \geq \alpha_{j}^{k}-\sigma_{l}^{k} \quad \forall k \in K,(j, l) \in A \\
0 \geq \alpha_{j}^{k}-\sigma_{j}^{k} \quad \forall k \in K, j \in I
\end{gathered}
$$

Proof. If we associate dual variables $\alpha_{j}^{k}$ to constraints (17), $\sigma_{l}^{k}$ to constraints (18) and $\beta_{j l}$ to constraints (19), by Farkas' Lemma, we get the result.

Notice that inequalities (11) form a subset of the projection inequalities of this hub location linearization. More precisely, inequalities (11) correspond to inequalities (21) for integer $(\alpha, \sigma, \beta) \geq 0$ which satisfy $\alpha_{j}^{k}=\sigma_{j}^{k}$ for all $k$ $\in K$ and $j \in I$ and $\beta_{j l}=\max _{k \in K}\left(\alpha_{j}^{k}-\sigma_{l}^{k}\right)$ for all $(j, l)$ $\in A$. However, remaining inequalities (21) are not necessarily dominated by inequalities (11). This is natural as the routing of the hub location is also feasible for the multicommodity formulation. 
Let $H$ be the cone of $(\alpha, \sigma, \beta) \geq 0$ that satisfy inequalities (22) and (23). Nondominated projection inequalities are among inequalities (21) that are defined by $(\alpha, \sigma, \beta)$, which are extreme rays of $H$ (see Balas [2]).

We consider first the case for a single commodity and characterize all nondominated inequalities. This gives us insight for the multicommodity case.

\subsection{Single Commodity Case}

Suppose $|K|=1$. We drop the index $k$ from the variables defined above.

Proposition 3. The ray $(\alpha, \sigma, \beta) \neq(0,0,0)$ is extreme for cone $H$ if and only if it belongs to one of the classes:

1. $\beta_{j l}=1$ for some $(j, l) \in A$ and the other entries are zero.

2. $\sigma_{j}=1$ for some $j \in I$ and the other entries are zero.

3. $\alpha_{j}=1$ for all $j \in S$, where $S \subseteq I, \sigma_{l}=1$ for all $l$ $\in I$ and the other entries are zero.

4. $\alpha_{j}=1$ for all $j \in S$ where $S \subseteq I, \sigma_{l}=1$ for all $l$ $\in T$ where $T \subseteq I$ such that $S \subseteq T, \beta_{j l}=1$ if $j \in S$, $l \notin T$ and the other entries are zero.

Proof. The proof is very similar to the proof of Proposition 3.5 given by Mirchandani [6]. We give it here for the sake of completeness.

(Sufficiency) Classes 1 and 2 are trivial. For classes 3 and 4 , consider $(\alpha, \sigma, \beta) \neq(0,0,0) \in H$. Let $B=\{(j$, $\left.l) \in A: \beta_{j l}>0\right\}, S=\left\{j \in I: \alpha_{j}>0\right\}$ and $T=\{l \in I$ : $\left.\sigma_{l}>0\right\}$. Notice that $S \subseteq T$ because of the constraint (23). Suppose that $(\alpha, \sigma, \beta)$ is not an extreme ray of $H$. Then there exist distinct $(\alpha, \sigma, \beta)^{1}$ and $(\alpha, \sigma, \beta)^{2}$ in $H$, which are not multiples of $(\alpha, \sigma, \beta)$ and $(\alpha, \sigma, \beta)=1 / 2(\alpha, \sigma, \beta)^{1}$ $+1 / 2(\alpha, \sigma, \beta)^{2}$. This implies that $\alpha_{j}^{1}=\alpha_{j}^{2}=0$ if $j \notin S$, $\sigma_{j}^{1}=\sigma_{j}^{2}=0$ if $j \notin T$ and $\beta_{j l}^{1}=\beta_{j l}^{2}=0$ if $(j, l) \notin B$.

In Class $3, B=\varnothing$ and $T=I$. As $\beta_{j l}^{1}=\beta_{j l}^{2}=0$ for all $(j, l)$ in $A, \sigma_{l}^{1} \geq \alpha_{j}^{1}$ and $\sigma_{l}^{2} \geq \alpha_{j}^{2}$ for all $j \in S$ and $l \in I$. As $\alpha_{j}^{1}+\alpha_{j}^{2}=2 \alpha_{j}=2$ and $\sigma_{l}^{1}+\sigma_{l}^{2}=2 \sigma_{l}=2$ we have that $\sigma_{l}^{1}=\alpha_{j}^{1}=\gamma^{1}$ and $\sigma_{l}^{2}=\alpha_{j}^{2}=\gamma^{2}$ for all $j \in S$ and $l \in I$. Then $(\alpha, \sigma, \beta)^{1}$ and $(\alpha, \sigma, \beta)^{2}$ are multiples of $(\alpha, \sigma$, $\beta)$.

In Class 4, $B, S$, and $T$ are all nonempty. If $j \in S$ and $l$ $\in T$, then $\beta_{j l}=0$. So we have $\sigma_{l}^{1} \geq \alpha_{j}^{1}$ and $\sigma_{l}^{2} \geq \alpha_{j}^{2}$ for all $j \in S$ and $l \in T$. By the above discussion, $\sigma_{l}^{1}=\alpha_{j}^{1}=$ $\gamma^{1}$ and $\sigma_{l}^{2}=\alpha_{j}^{2}=\gamma^{2}$ for all $j \in S$ and $l \in T$.

If $j \in S$ and $l \notin T$, then $\beta_{j l}^{1} \geq \alpha_{j}^{1}$ and $\beta_{j l}^{2} \geq \alpha_{j}^{2}$. As $\alpha_{j}^{1}$ $+\alpha_{j}^{2}=2 \alpha_{j}=2$ and $\beta_{j l}^{1}+\beta_{j l}^{2}=2 \beta_{j l}=2$, we get $\beta_{j l}^{1}$ $=\alpha_{j}^{1}=\gamma^{1}$ and $\beta_{j l}^{2}=\alpha_{j}^{2}=\gamma^{2}$. So $(\alpha, \sigma, \beta)^{1}$ and $(\alpha, \sigma$, $\beta)^{2}$ are multiples of $(\alpha, \sigma, \beta)$.

For classes 3 and 4 , we showed that a ray $(\alpha, \sigma, \beta)$ satisfying the requirements of one of these classes cannot be written as a linear combination of two distinct rays of $H$. Thus, such a ray $(\alpha, \sigma, \beta)$ is an extreme ray.

(Necessity) Given an extreme ray $(\alpha, \sigma, \beta) \neq(0,0,0)$ of
$H$, define the sets $B, S$, and $T$. Assume that $S=\varnothing$. It is easy to show that if $T=\varnothing$, then $(\alpha, \sigma, \beta)$ should belong to class 1 and if $B=\varnothing$ then $(\alpha, \sigma, \beta)$ should belong to class 2. If both $T$ and $B$ are not empty, then this can be written as a linear combination of rays of classes 1 and 2, so it cannot be extreme.

Now, assume that $S \neq \varnothing$. By feasibility, we have $S \subseteq$ $T$. Define $\gamma=\min \left\{\min _{j \in S} \alpha, \min _{l \in T} \sigma_{l}\right\}$. Clearly, $\gamma>0$. Consider the two rays $(\alpha, \sigma, \beta)^{1}$ and $(\alpha, \sigma, \beta)^{2}$ defined as follows: $\alpha_{j}^{1}=\gamma$ for all $j \in S, \sigma_{l}^{1}=\gamma$ for all $l \in T, \beta_{j l}^{1}$ $=\gamma$ for all $j \in S, l \notin T, \alpha_{j}^{2}=2 \alpha_{j}-\gamma$ for all $j \in S, \sigma_{l}^{2}$ $=2 \sigma_{l}-\gamma$ for all $l \in T, \beta_{j l}^{2}=2 \beta_{j l}-\gamma$ for all $j \in S$, $l \notin T, \beta_{j l}^{2}=2 \beta_{j l}$ for all $j \in S, l \in T$ or $j \notin S$ and the rest of entries are 0 . Both $(\alpha, \sigma, \beta)^{1}$ and $(\alpha, \sigma, \beta)^{2}$ are in $H$ and $(\alpha, \sigma, \beta)=1 / 2(\alpha, \sigma, \beta)^{1}+1 / 2(\alpha, \sigma, \beta)^{2}$. But as $(\alpha, \sigma$, $\beta)$ is an extreme ray, $(\alpha, \sigma, \beta)^{1}$ and $(\alpha, \sigma, \beta)^{2}$ should be multiples of $(\alpha, \sigma, \beta)$. So $\alpha_{j}=1$ for all $j \in S, \sigma_{l}=1$ for all $l \in T, \beta_{j l}=1$ for all $j \in S, l \notin T$ and the rest of the entries are 0 .

If $B=\varnothing$, by feasibility we have $T=I$. Then $(\alpha, \sigma, \beta)$ is in Class 3. Otherwise, it is in Class 4.

Proposition 4. Given ( $x, z)$ which satisfies (1), (2), (4), and (5), there exists $X$ that satisfies (17)-(20) if and only if

$$
\sum_{(j, l) \in A: j \in S, l \notin T} z_{j l} \geq t\left(\sum_{j \in S} x_{o j}-\sum_{l \in T} x_{d l}\right)
$$

for all $S \subseteq T \subseteq I$.

Proof. Inequalities (21) defined by $(\alpha, \sigma, \beta)$ that are extreme rays of $H$ are as follows:

1. $z_{j l} \geq 0$ for all $(j, l) \in A$.

2. $x_{d j} \geq 0$ for all $j \in I$.

3. $\sum_{j \in S} x_{o j} \leq 1$ for all $S \subseteq I$

4. $\sum_{(j, l) \in A: j \in S, l \notin T} z_{j l} \geq t\left(\sum_{j \in S} x_{o j}-\sum_{l \in T} x_{d l}\right)$ for all $S$ $\subseteq T \subseteq I$.

The first three families of inequalities are implied by constraints (5), (4), and (1), respectively. The only nonredundant inequalities are the inequalities of the fourth form.

These inequalities are quite similar to cut inequalities for the single commodity flow. In fact, when we take a cut in the case of hub location, we choose two disjoint subsets of the set $I, S$ and $\bar{T}=\Lambda T$ and consider all the arcs going from $S$ to $\bar{T}$.

Proposition 5. Given $(x, z)$, we can separate inequalities (24) by solving a min cut problem.

Proof. Separation of (24) is to find $S \subseteq T \subseteq I$ such that $\sum_{(j, l) \in A: j \in S, l \notin T} \quad z_{j l}-t\left(\sum_{j \in S} x_{o j}-\sum_{l \in T} x_{d l}\right)$ is minimized. Let $\phi$ denote this minimum value. Consider the layered graph $G^{\prime}=\left(V, A^{\prime}\right)$ where $V$ includes the nodes $O$ and $d$, the set $I$ and a duplicate $I^{\prime}$ of set $I$, that is, $V=\{o$, 


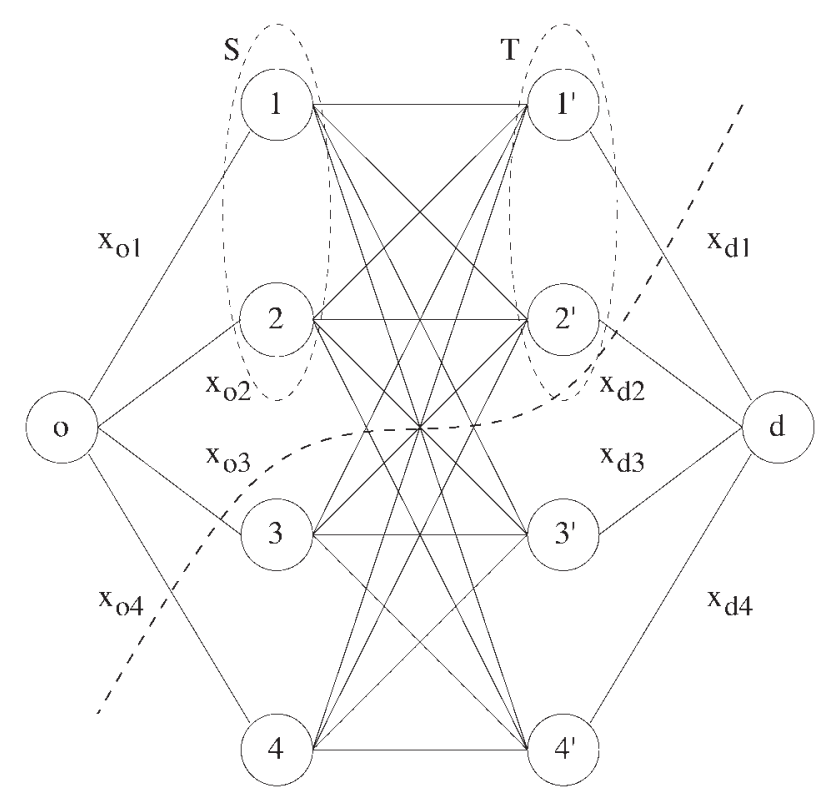

FIG. 2. The separation problem as a min cut.

d) $\cup I \cup I^{\prime}$. The arc set is $A^{\prime}=\{(o, j): j \in I\} \cup\{(j$, $\left.l): j \in I, l \in I^{\prime}\right\} \cup\left\{(l, d): l \in I^{\prime}\right\}$. Let $w_{i j}$ denote the capacity of $\operatorname{arc}(i, j) \in A^{\prime}$ defined as follows:

$$
\begin{gathered}
w_{o j}=t x_{o j} \quad \forall j \in I \\
w_{l d}=t x_{d l} \quad \forall l \in I^{\prime} \\
w_{j l}=z_{j l} \quad \forall j \in I, l \in I^{\prime}:(j, l) \in A \\
w_{j j^{\prime}}=\infty \quad \forall j \in I, j^{\prime} \in I^{\prime}
\end{gathered}
$$

A cut set $C$ is a subset of the set $V$ such that $o \in C$ and $d$ $\notin C$. Define $S=C \cap I$ and $T=C \cap I^{\prime}$. If the duplicates of nodes in $S$ are not in $T$, the cut has an infinite capacity. Otherwise, the capacity of cut set $C$ is

$$
\begin{aligned}
\sum_{(i, j) \in \delta(C)} w_{i j}=\sum_{j \notin S} t x_{o j}+ & \sum_{j \in S} \sum_{l \notin T} z_{j l}+\sum_{l \in T} t x_{d l} \\
& =t-\sum_{j \in S} t x_{o j}+\sum_{l \in T} t x_{d l}+\sum_{j \in S} \sum_{l \notin T} z_{j l}
\end{aligned}
$$

As cut set $C=\{o\}$ has capacity $t$, the min cut problem has a finite value. So, $\phi=\min _{C} \sum_{(i, j) \in \delta(C)} w_{i j}-t$.

In Figure 2, the set $C=\left\{o, 1,2,1^{\prime}, 2^{\prime}\right\}$. So $S=\{1$, $2\}, T=\left\{1^{\prime}, 2^{\prime}\right\}$ and the corresponding inequality is:

$$
z_{13}+z_{14}+z_{23}+z_{24} \geq t\left(x_{o 1}+x_{o 2}-x_{d 1}-x_{d 2}\right)
$$

\subsection{Multicommodity Case}

Now we consider the multicommodity case. For $(\alpha, \sigma, \beta)$ $\neq(0,0,0)$ in $H$, define $B=\left\{(j, l) \in A: \beta_{j l}>0\right\}, S_{k}$ $=\left\{j \in J: \alpha_{j}^{k}>0\right\}$ and $T_{k}=\left\{l \in I: \sigma_{l}^{k}>0\right\}$ for all $k \in K$ and $K^{\prime}=\left\{k \in K: S_{k} \neq \varnothing\right\}$.

The extreme rays for which $B=\varnothing$ or $K^{\prime}=\varnothing$ are characterized as follows:

1. If $S_{k}=T_{k}=\varnothing$ for all $k \in K$, then $(\alpha, \sigma, \beta)$ is an extreme ray if and only if $\beta_{j l}=1$ for some $(j, l) \in A$ and the rest of entries are zero. The corresponding projection inequality is $z_{j l} \geq 0$.

2. If $S_{k}=\varnothing$ for all $k \in K$ and $B=\varnothing$, then $(\alpha, \sigma, \beta)$ is an extreme ray if and only if $\sigma_{j}^{k}=1$ for some $j \in I$ and for some $k \in K$ and the rest of the entries are 0 . The corresponding projection inequality is $x_{d(k) j} \geq 0$.

3. If $B=\varnothing$ and $S_{k} \subseteq I$ for all $k \in K^{\prime}$, then we should have $T_{k}=I$ for all $k \in K^{\prime}$. In this case, $(\alpha, \sigma, \beta)$ is an extreme ray if and only if $\left|K^{\prime}\right|=1$. The corresponding projection inequality is $\sum_{j \in S_{k}} x_{o(k) j} \leq 1$.

These propositions can be proved in a similar way to the proof of Proposition 3.

We have a sufficient condition for a special class of the remaining rays to be extreme.

Proposition 6. (Labbé et al. [5]) Let $S_{k} \subseteq T_{k} \subseteq$ I for all $k$ $\in K$. Define $(\alpha, \sigma, \beta)$ such that

$$
\begin{array}{cl}
\alpha_{j}^{k}=1 & j \in S_{k} \\
\sigma_{l}^{k}=1 & l \in T_{k}
\end{array}
$$

$\beta_{j l}=1 \quad$ if there exists a $k \in K$ such that $j \in S_{k}$ and $l \notin T_{k}$

and the other entries are 0 . Then $(\alpha, \sigma, \beta) \in H$.

Define $G^{\prime}=(B, E)$ where $B=\left\{(j, l) \in A: \beta_{j l}=1\right\}$ and $E=\left\{\{(j, l),(m, n)\}:(j, l) \in B,(m, n) \in B, \beta_{j l}=\alpha_{j}^{k}\right.$ and $\beta_{m n}=\alpha_{m}^{k}$ for some $\left.k \in K\right\}$. For $k \in K$, define also the bipartite graph $G_{k}^{\prime}=\left(S_{k} \times T_{k}, E_{k}^{\prime}\right)$ where $E_{k}^{\prime}=\{\{j, l\}: j$ $\in S_{k}, l \in T_{k}, \beta_{j l}=0$ or $\left.j=l\right\}$.

Ray $(\alpha, \sigma, \beta)$ is extreme if graphs $G^{\prime}$ and $G_{k}^{\prime}$ are connected for all $k \in K$ and $S_{k} \neq I$ for all $k \in K$.

A special class of these extreme rays define inequalities that are similar to inequalities (24).

Proposition 7. (Labbé et al. [5]) The inequality

$$
\sum_{j \in S} \sum_{l \in T} z_{j l} \geq \sum_{k \in K^{\prime}} t^{k}\left[\sum_{j \in S} x_{o(k) j}+\sum_{l \in T} x_{d(k) l}-1\right]
$$

where $S$ and $T$ are nonempty disjoint subsets of $I$ and $K^{\prime} \subseteq$ $K$ is a valid inequality, and it is not dominated by other projection inequalities.

Moreover, a subset of these inequalities are indeed sufficient to have a formulation of UHL. 
Proposition 8. (Labbé et al. [5]) For ( $x, z)$ which satisfies (1), (2), (4), and (5), there exists $X$ that satisfies (17)-(20) if and only if $(x, z)$ satisfies inequalities

$$
z_{j l} \geq \sum_{k \in K^{\prime}} t^{k}\left(x_{o(k) j}+x_{d(k) l}-1\right) \quad \forall K^{\prime} \subseteq K,(j, l) \in A
$$

For the single commodity case, these inequalities simplify to

$$
\begin{gathered}
z_{j l} \geq t\left(x_{o j}+x_{d l}-1\right) \quad \forall(j, l) \in A \\
z_{j l} \geq 0 \quad \forall(j, l) \in A
\end{gathered}
$$

This corresponds to the classical way of linearizing constraints $z_{j l}=t x_{o j} x_{d l}$ for all $(j, l) \in A$ when we minimize a cost function where variables $z_{j l}$ s have positive coefficients.

\section{FACETS OF THE UHL POLYHEDRON}

Polyhedral properties of UHL are studied in Labbé et al. [5]. Here we summarize their results and present some new facet defining inequalities.

We replace constraints (3) by (26) to have a linear formulation. We also eliminate the variables $x_{j j}$ s by substituting $x_{j j}=1-\sum_{m \in \Lambda\{j\}} x_{j m}$ for all $j \in I$ (see Avella and Sassano [1]).

If both $j$ and $l$ become hubs, then the traffic of commodities with destination $j$ or origin $l$ does not travel on $\operatorname{arc}(j$, $l$ ). Moreover, the traffic from node $j$ to node $l$ travels on arc $(j, l)$. Define $K_{j l}=K \backslash(\{(j, l)\} \cup\{(m, j): m \in \Lambda \backslash\{j\}\}$ $\cup\{(l, m): m \in \Lambda\{l\}\})$.

The UHL can be reformulated as follows:

$$
\begin{gathered}
\min \sum_{i \in I} \sum_{j \in \Lambda\{i\}\}} F_{i j} x_{i j}+\sum_{i \in I} F_{i i}\left(1-\sum_{j \in \Lambda\{i\}} x_{i j}\right)+\sum_{(j, l) \in A} R_{j l} z_{j l} l \\
\text { s.t. } x_{i j}+\sum_{m \in \Lambda\{j\}} x_{j m} \leq 1 \quad \forall(i, j) \in A \\
z_{j l} \geq \sum_{k \in K^{\prime}: o(k) \neq j, d(k) \neq e} t^{k}\left(x_{o(k) j}+x_{d(k) l}-1\right) \\
+\sum_{i \in \Lambda\{j, l\}:(j, i) \in K^{\prime}} t_{j i}\left(x_{i l}-\sum_{m \in N\{j\}} x_{j m}\right)+\sum_{i \in \Lambda\{j, l\}:(i, l) \in K^{\prime}} t_{i l}\left(x_{i j}\right. \\
\left.-\sum_{m \in \Lambda\{\{\}} x_{l m}\right)+t_{j l}\left(1-\sum_{m \in N\{j\}} x_{j m}-\sum_{m \in \Lambda\{l\}} x_{l m}\right) \\
\quad \forall K^{\prime} \subseteq K_{j l},(j, l) \in A \\
x_{i j} \in\{0,1\} \quad \forall(i, j) \in A \\
z_{j l} \geq 0 \quad \forall(j, l) \in A
\end{gathered}
$$

Let $P_{U H}=\operatorname{conv}\left(\left\{(x, z) \in\{0,1\}^{n(n-1)} \times \mathbb{R}^{n(n-1)}:(x\right.\right.$, z) satisfies (27)-(30)\}). Define $e_{i j}^{x}=(x, z)$ (resp. $e_{i j}^{z}=(x$, $z))$ to be the unit vector such that $x_{l m}=0$ for all $(l, m)$ $\in A \backslash\{(i, j)\}, x_{i j}=1$ and $z_{l m}=0$ for all $(l, m) \in A$ (resp. $x_{l m}=0$ for all $(l, m) \in A, z_{l m}=0$ for all $(l, m) \in A \backslash\{(i$, $j)\}$ and $\left.z_{i j}=1\right)$.

Proposition 9. (Labbé et al. [5]) The polyhedron $P_{U H}$ is full dimensional, that is, $\operatorname{dim}\left(P_{U H}\right)=2 n(n-1)$.

Theorem 1. (Labbé et al. [5]) The inequality $\pi x \leq \pi_{0}$ defines a facet of $P_{U H}$ if and only if it defines a facet of $P_{U C}$ $=\operatorname{conv}\left\{x \in\{0,1\}^{n(n-1)}: x_{i j}+\sum_{m \in \Lambda\{j\}} x_{j m} \leq 1 \forall(i, j)\right.$ $\in A\}$.

The polytope $P_{U C}$ is a special stable set polytope. For facet defining inequalities of $P_{U C}$, see Yaman [10].

Theorem 2. (Labbé et al. [5]) No inequality of the form $\beta z$ $\geq \beta_{0}$ defines a facet of $P_{U H}$ unless it is a positive multiple of $z_{j l} \geq 0$ for some $(j, l) \in A$.

Proposition 10. (Labbé et al. [5]) For $(j, l) \in A$, if $t_{j l}=0$, then the inequality $z_{j l} \geq 0$ defines a facet of $P_{U H}$.

The remaining facet defining inequalities of $P_{U H}$ involve both $x$ and $z$ variables. Here, we investigate which inequalities (28) are facet defining inequalities.

Proposition 11. For $(j, l) \in A$ and $K^{\prime} \subseteq K_{j l}$, define $I_{j}^{\prime}$ $=\left\{m \in \Lambda\{l\}: \exists i \in \Lambda\{m\}:(i, m) \in K^{\prime}, t_{i m}>0\right\}$ and $I_{l}^{\prime}$ $=\left\{m \in \Lambda\{j\}: \exists i \in \Lambda\{m\}:(m, i) \in K^{\prime}, t_{m i}>0\right\}$. If inequality (28) defines a facet of $P_{U H}$, then $I_{j}^{\prime}=I_{l}^{\prime}=\varnothing$.

Proof. Assume that $(x, z) \in P_{U H}$ satisfies inequality (28) at equality and that $x_{j m}=1$ for some $m \in I_{j}^{\prime}$. Then the right-hand side of inequality (28) is

$$
\begin{aligned}
& \sum_{k \in K^{\prime}: o(k) \neq j, d(k) \neq e} t^{k}\left(x_{d(k) l}-1\right)+\sum_{i \in \Lambda\{j, l]:(j, i) \in K^{\prime}} t_{j i}\left(x_{i l}-1\right) \\
& -\sum_{i \in \Lambda\left\{j, l:(i, l) \in K^{\prime}\right.} t_{i l} \sum_{m \in \Lambda\{l\}} x_{l m}-t_{j l} \sum_{m \in \Lambda\{l\}} x_{l m} \\
& \leq \sum_{k \in K^{\prime}: o(k) \neq j, d(k) \neq e} t^{k}\left(x_{d(k) l}-1\right)+\sum_{i \in \Lambda\{j, l\}:(j, i) \in K^{\prime}} t_{j i}\left(x_{i l}-1\right) \\
& \quad \leq \sum_{i \in \Lambda\{m\}:(i, m) \in K^{\prime}} t_{i m}\left(x_{m l}-1\right)=-\sum_{i \in \Lambda\{m\}:(i, m) \in K^{\prime}} t_{i m}<0
\end{aligned}
$$

So, any $(x, z) \in P_{U H}$ that satisfies inequality (28) at equality also satisfies $x_{j m}=0$ for $m \in I_{j}^{\prime}$. We can show that $(x, z)$ should also satisfy $x_{l m}=0$ for $m \in I_{l}^{\prime}$ in a similar way. So if at least one of sets $I_{j}^{\prime}$ and $I_{l}^{\prime}$ is nonempty, then inequality (28) is not facet defining for $P_{U H}$.

If $t^{k}=0$ for all $k \in K^{\prime}$, then both sets $I_{j}^{\prime}$ and $I_{l}^{\prime}$ are empty. We next show that in this case, inequality (28) is facet defining. Define $N$ to be a very large integer. 
Proposition 12. For $(j, l) \in A$, if $t^{k}=0$ for all $k \in K^{\prime}$, then inequality (28) is facet defining for $P_{U H}$.

Proof. Assume that $t^{k}=0$ for all $k \in K^{\prime}$. Below are $2 n(n-1)$ affinely independent points in $P_{U H}$ that satisfy inequality (28) at equality:

- $\Sigma_{(t, s) \in A \backslash\{(j, l)\}} N e_{t s}^{z}+t_{j l} e_{j l}^{z}$

- $\sum_{(t, s) \in A \backslash\{(j, l)\}} N e_{t s}^{z}+t_{j l} e_{j l}^{z}+e_{i m}^{z}$ for $(i, m) \in A \backslash\{(j$, l) $\}$

- $e_{i m}^{x}+\sum_{(t, s) \in A \backslash\{(j, l)\}} N e_{t s}^{z}+t_{j l} e_{j l}^{z}$ for $i \in \Lambda\{j, l\}$ and $m \in \Lambda\{i, j, l\}$

- $e_{j m}^{x}+\Sigma_{(t, s) \in A \backslash\{(j, l)\}} N e_{t s}^{z}$ for $m \in \Lambda\{j\}$

- $e_{l m}^{x}+\Sigma_{(t, s) \in A \backslash\{(j, l)\}} N e_{t s}^{z}$ for $m \in \Lambda\{l\}$

- $e_{i j}^{x}+e_{l j}^{x}+\Sigma_{(t, s) \in A \backslash\{(j, l)\}} N e_{t s}^{z}$ for $i \in \Lambda\{j, l\}$

- $e_{i l}^{x}+e_{j l}^{x}+\Sigma_{(t, s) \in A \backslash\{(j, l)\}} N e_{t s}^{z}$ for $i \in \Lambda\{j, l\}$.

In the sequel, we present two more families of facetdefining inequalities that dominate some projection inequalities.

Proposition 13. For $(j, l) \in A$, the inequality

$$
\begin{array}{r}
z_{j l} \geq t_{j l}\left(1-\sum_{i \in \Lambda\{j\}} x_{j i}-\sum_{i \in \Lambda\{l\}} x_{l i}\right)+\sum_{i \in \Lambda\{j, l\}} t_{j i}\left(x_{i l}-\sum_{m \in \Lambda\{i, j\}} x_{j m}\right) \\
+\sum_{i \in \Lambda\{j, l\}} t_{i l}\left(x_{i j}-\sum_{m \in \Lambda\{i, l\}} x_{l m}\right)
\end{array}
$$

is valid for $P_{U H}$.

Proof. If $x_{j i}=x_{l i}=0$ for all $i \in \Lambda\{j, l\}$, then inequality (31) is the same as inequality (28) for $K^{\prime}=\{(j$, i) $: i \in \Lambda\{j, l\}\} \cup\{(i, l): i \in \Lambda\{j, l\}\}$. If $x_{j p}=1$ for some $p \in \Lambda\{j, l\}$, then the right-hand side of inequality (31) is

$$
-t_{j l} \sum_{i \in \Lambda\{l\}} x_{l i}+\sum_{i \in \Lambda\{j, l, p\}} t_{j i}\left(x_{i l}-1\right)-\sum_{i \in \Lambda\{j, l\}} t_{i l} \sum_{m \in \Lambda\{i, l\}} x_{l m} \leq 0
$$

The case where $x_{l p}=1$ for some $p \in \Lambda\{j, l\}$ is analogous.

The proof of Proposition 13 shows that inequality (31) dominates inequality (28) for $K^{\prime}=\{(j, i): i \in \Lambda\{j, l\}\}$ $\cup\{(i, l): i \in \Lambda\{j, l\}\}$.

Proposition 14. For $(j, l) \in A$, inequality (31) defines a facet of $P_{U H}$.

Proof. Below are $2 n(n-1)$ affinely independent points in $P_{U H}$ that satisfy inequality (31) at equality:

- $\Sigma_{(t, s) \in A \backslash\{(j, l)\}} N e_{t s}^{z}+t_{j l} l_{j l}^{z}$

- $\Sigma_{(t, s) \in A \backslash\{(j, l)\}} N e_{t s}^{z}+t_{j l} e_{j l}^{z}+e_{i m}^{z}$ for $(i, m) \in A \backslash\{(j$, l) $\}$

- $e_{i m}^{x}+\sum_{(t, s) \in A \backslash\{(j, l)\}} N e_{t s}^{z}+t_{j l} e_{j l}^{z}$ for $i \in \Lambda\{j, l\}$ and $m \in \Lambda\{i, j, l\}$
- $e_{j m}^{x}+\sum_{i \in \Lambda\{j, l, m\}} e_{i l}^{x}+\sum_{(t, s) \in A \backslash\{(j, l)\}} N e_{t s}^{z}$ for $m$ $\in \Lambda\{j\}$

- $e_{l m}^{x}+\sum_{i \in \Lambda\{j, l, m\}} e_{i j}^{x}+\sum_{(t, s) \in A \backslash\{(j, l)\}} N e_{t s}^{z}$ for $m$ $\in \Lambda\{l\}$

- $e_{i j}^{x}+\Sigma_{(t, s) \in A \backslash\{(j, l)\}} N e_{t s}^{z}+\left(t_{j l}+t_{i l}\right) e_{j l}^{z}$ for $i \in \Lambda\{j$, l\}

- $e_{i l}^{x}+\sum_{(t, s) \in A \backslash\{(j, l)\}} N e_{t s}^{z}+\left(t_{j l}+t_{j i}\right) e_{j l}^{z}$ for $i \in \Lambda\{j$, $l\}$.

Proposition 15. For $(j, l) \in A, I_{j} \subseteq \Lambda\{j, l\}$ and $I_{l} \subseteq \Lambda\{j$, l\} such that $I_{j} \cap I_{l}=\varnothing$ and $I_{j} \cup I_{l}=\Lambda\{j$, l\}, the inequality

$$
\begin{array}{r}
z_{j l} \geq \sum_{i \in I_{j}} \sum_{m \in I_{l}} t_{i m}\left(x_{i j}+x_{m l}+x_{i m}+x_{m i}-1\right) \\
+\sum_{i \in I_{l}} t_{j i}\left(x_{i l}-\sum_{m \in \Lambda\{i, j\}} x_{j m}\right)+\sum_{i \in I_{j}} t_{i l}\left(x_{i j}-\sum_{m \in \Lambda\{i, l\}} x_{l m}\right) \\
+t_{j l}\left(1-\sum_{i \in \Lambda\{j\}} x_{j i}-\sum_{i \in \Lambda\{l\}} x_{l i}\right)
\end{array}
$$

is valid for $P_{U H}$.

Proof. Assume that $x_{i m}=x_{m i}=0$ for all $i \in I_{j}$ and $m \in I_{l}$ and $x_{j m}=x_{l m}=0$ for all $m \in \Lambda\{j, l\}$. Then inequality (32) is the same as inequality (28) for $K^{\prime}=\{(i$, $\left.m) \in K: i \in I_{j}, m \in I_{l}\right\} \cup\left\{(i, l) \in K: i \in I_{j}\right\} \cup\{(j$, i) $\left.\in K: i \in I_{l}\right\}$ and is valid. If $x_{j m}=1$ for some $m$ $\in \Lambda\{j\}$ then we can show that inequality (32) is still valid as in the proof of Proposition 13. So inequality (32) is valid if $x_{i m}=x_{m i}=0$ for all $i \in I_{j}$ and $m \in I_{l}$. Notice that if $x_{i m}+x_{m i}=1$ for some $i \in I_{j}$ and $m \in I_{l}$ then $x_{i j}+x_{m l}$ $=0$. So inequality (32) is valid for $P_{U H}$.

Inequality (32) dominates inequality (28) for $K^{\prime}=\{(i$, $\left.m) \in K: i \in I_{j}, m \in I_{l}\right\} \cup\left\{(i, l) \in K: i \in I_{j}\right\} \cup\{(j$, i) $\left.\in K: i \in I_{l}\right\}$.

Proposition 16. For $(j, l) \in A, I_{j} \subseteq \Lambda\{j, l\}$ and $I_{l} \subseteq \Lambda\{j$, l) such that $I_{j} \cap I_{l}=\varnothing$ and $I_{j} \cup I_{l}=\Lambda\{j, l\}$, inequality (32) defines a facet of $P_{U H}$.

Proof. Below are $2 n(n-1)$ affinely independent points in $P_{U H}$ that satisfy inequality (32) at equality:

- $\sum_{p \in I_{j}} e_{p j}^{x}+\sum_{(t, s) \in A \backslash\{(j, l)\}} N e_{t s}^{z}+\sum_{p \in I_{j} \cup\{j\}} t_{p l} e_{j l}^{z}$

- $\sum_{p \in I_{j}} e_{i j}^{x}+\sum_{(t, s) \in A \backslash\{(j, l)\}} N e_{t s}^{z}+\sum_{p \in I_{j} \cup\{j\}} t_{p l} e_{j l}^{z}+$ $e_{i m}^{z}$ for $(i, m) \in A \backslash\{(j, l)\}$

- $e_{i m}^{x}+\sum_{\left.p \in I_{Y} \backslash m\right\}} e_{p l}^{x}+\Sigma_{\left.p \in I_{j} \backslash i\right\}} e_{p j}^{x}+\Sigma_{(t, s) \in A \backslash\{(j, l)\}}$ $N e_{t s}^{z}+\sum_{p \in I_{j} \backslash\{i\} \cup\{j\}} \sum_{r \in I_{I}\{m\} \cup\{l\}} t_{p r} e_{j l}^{z}$ for $i \in I_{j}$ and $m \in I_{l}$

- $e_{m i}^{x}+\sum_{p \in I_{l} \backslash\{m\}} e_{p l}^{x}+\sum_{\left.p \in I_{\backslash} \backslash i\right\}} e_{p j}^{x}+\sum_{(t, s) \in A \backslash\{(j, l)\}}$ $N e_{t s}^{z}+\sum_{p \in I_{i \backslash\{i\}} \cup\{j\}} \sum_{\left.r \in I_{\backslash} \backslash m\right\} \cup\{l\}} t_{p r} e_{j l}^{z}$ for $i \in I_{j}$ and $m \in I_{l}$

- $e_{i m}^{x}+\sum_{p \in I_{l}} e_{p l}^{x}+\sum_{(t, s) \in A \backslash\{(j, l)\}} N e_{t s}^{z}+\Sigma_{p \in I_{l} \cup\{l\}}$ $t_{j p} e_{j l}^{z}$ for $i \in I_{j}$ and $m \in I_{j}$

- $e_{i m}^{x}+\sum_{p \in I_{i}} e_{p j}^{x}+\sum_{(t, s) \in A \backslash\{(j, l)\}} N e_{t s}^{z}+\sum_{p \in I_{i} \cup\{j\}}$ $t_{p l} e_{j l}^{z}$ for $i \in I_{l}$ and $m \in I_{l}$

- $e_{i j}^{x}+\sum_{p \in I_{l}} e_{p l}^{x}+\sum_{(t, s) \in A \backslash\{(j, l)\}} N e_{t s}^{z}+\sum_{p \in I_{l} \cup\{l\}}\left(t_{i p}\right.$ $\left.+t_{j p}\right) e_{j l}^{z}$ for $i \in I_{j}$ 
- $e_{i l}^{x}+\sum_{p \in I_{l}} e_{p l}^{x}+e_{j l}^{x}+\sum_{(t, s) \in A \backslash\{(j, l)\}} N e_{t s}^{z}$ for $i \in I_{j}$

- $e_{i l}^{x}+\sum_{p \in I_{j}} e_{p j}^{x}+\sum_{(t, s) \in A \backslash\{(j, l)\}} N e_{t s}^{z}+\sum_{p \in I_{j} \cup\{j\}}\left(t_{p i}\right.$ $\left.+t_{p l}\right) e_{j l}^{z}$ for $i \in I_{l}$

- $e_{i j}^{x}+\sum_{p \in I_{j}} e_{p j}^{x}+e_{l j}^{x}+\sum_{(t, s) \in A \backslash\{(j, l)\}} N e_{t s}^{z}$ for $i \in I_{l}$

- $e_{j i}^{x}+\sum_{p \in I_{l}} e_{p l}^{x}+\sum_{(t, s) \in A \backslash\{(j, l)\}} N e_{t s}^{z}$ for $i \in I_{j} \cup\{l\}$

- $e_{j i}^{x}+\sum_{p \in I_{l} \backslash\{i\}} e_{p l}^{x}+\sum_{p \in I_{j}} e_{p i}^{x}+\sum_{(t, s) \in A \backslash\{(j, l)\}} N e_{t s}^{z}$ for $i \in I_{l}$

- $e_{l i}^{x}+\sum_{p \in I_{j}} e_{p j}^{x}+\sum_{(t, s) \in A \backslash\{(j, l)\}} N e_{t s}^{z}$ for $i \in I_{l} \cup\{j\}$

- $e_{l i}^{x}+\sum_{p \in I_{j} \backslash\{i\}} e_{p j}^{x}+\sum_{p \in I_{l}} e_{p i}^{x}+\sum_{(t, s) \in A \backslash\{(j, l)\}} N e_{t s}^{z}$ for $i \in I_{j}$.

Before concluding this section, we discuss the separation problems related to inequalities (28), (31), (32), and (33). For $(j, l) \in A$, inequalities (28) can be separated in polynomial time by taking

$$
\begin{array}{r}
K^{\prime}=\left\{(i, m) \in K_{j l}: i, m \in \Lambda\{j, l\}, x_{o(k) j}+x_{d(k) l}\right. \\
>1\} \cup\left\{i \in \Lambda\{j, l\}: x_{i l}>\sum_{m \in \Lambda\{j\}} x_{j m}\right\} \cup\left\{i \in \Lambda\{j, l\}: x_{i j}\right. \\
\left.>\sum_{m \in \Lambda\{l\}} x_{l m}\right\}
\end{array}
$$

Inequalities (31) can also be separated in polynomial time by enumeration. However, the separation of inequalities (32) seems more complicated. In fact, this separation problem is a special max cut problem on a graph $G_{c}=(I$, $\left.A_{c}\right)$. Let $w_{a}$ denote the capacity of arc $a \in A_{c}$. The arcs and their capacities are defined as follows:

$$
\begin{array}{ll}
(j, i): i \in \Lambda\{j, l\} & w_{j i}=t_{j i}\left(x_{i l}-\sum_{m \in \Lambda\{i, j\}} x_{j m}\right) \\
(i, l): i \in \Lambda\{j, l\} & w_{i l}=t_{i l}\left(x_{i j}-\sum_{m \in \Lambda\{i, l\}} x_{l m}\right)
\end{array}
$$$$
(i, m): i, m \in \Lambda\{j, l\}
$$

$$
\begin{aligned}
w_{i m} & =t_{i m}\left(x_{i j}+x_{m l}+x_{i m}+x_{m i}-1\right) \\
(j, l) \quad w_{j l} & =t_{j l}\left(1-\sum_{i \in \Lambda\{j\}} x_{j i}-\sum_{i \in \Lambda\{l\}} x_{l i}\right)
\end{aligned}
$$

The capacity of a max cut separating $j$ and $l$ in $G_{c}$ is equal to the maximum value that the right-hand side of inequality (32) can attain. So for $(j, l) \in A$, the most violated inequality (32) can be found by solving a max cut problem.

We now present a family of valid inequalities that generalizes inequalities (32) and that can be separated in polynomial time.

Proposition 17. For $(j, l) \in A$ and $K^{\prime} \subseteq K_{j l}$, the inequality

$z_{j l} \geq \sum_{k \in K^{\prime}: o(k) \neq j, d(k) \neq e} t^{k}\left(x_{o(k) j}+x_{d(k) l}+x_{o(k) d(k)}+x_{d(k) o(k)}-1\right)$

$$
\begin{gathered}
+\sum_{i \in \Lambda\{j, l\}:(j, i) \in K^{\prime}} t_{j i}\left(x_{i l}-\sum_{m \in \Lambda\{i, j\}} x_{j m}\right)+\sum_{i \in \Lambda\{j, l\}:(i, l) \in K^{\prime}} t_{i l}\left(x_{i j}\right. \\
\left.\quad-\sum_{m \in \Lambda\{i, l\}} x_{l m}\right)+t_{j l}\left(1-\sum_{m \in \Lambda\{j\}} x_{j m}-\sum_{m \in \Lambda\{l\}} x_{l m}\right)
\end{gathered}
$$

is valid for $P_{U H}$.

Proof. Similar to the proof of Proposition 15.

Clearly, for a given $(j, l) \in A$ and $K^{\prime} \subseteq K_{j l}$, inequality (33) dominates inequality (28). Propositions 12 and 17 imply the following:

Corollary 1. For $(j, l) \in A$ and $K^{\prime} \subseteq K_{j l}$, inequality (28) is facet defining for $P_{U H}$ if and only if $t^{k}=0$ for all $k \in K^{\prime}$.

Inequalities (33) can be separated in polynomial time by taking for $(j, l) \in A$,

$$
\begin{gathered}
K^{\prime}=\left\{(i, m) \in K_{j l}: i, m \in \Lambda\{j, l\}, x_{o(k) j}+x_{d(k) l}+x_{o(k) d(k)}\right. \\
\left.+x_{d(k) o(k)}>1\right\} \cup\left\{i \in \Lambda\{j, l\}: x_{i l}>\sum_{m \in \Lambda\{i, j\}} x_{j m}\right\} \\
\cup\left\{i \in \Lambda\{j, l\}: x_{i j}>\sum_{m \in \Lambda\{i, l\}} x_{l m}\right\}
\end{gathered}
$$

In the example below, we show that using inequalities (33), we can cut some fractional solutions that do not violate inequalities (28).

Example 1. Assume that $I=\{1,2,3,4\}$ and the only nonzero traffic demand is from node 3 to node 4 and $t_{34}=1$. Consider $(x, z)$ such that $x_{31}=x_{42}=x_{34}=0.5$, the remaining entries of $x$ are zero and $z=0$. The vector $(x, z)$ satisfies all inequalities (28). Inequality $(33)$ for $\operatorname{arc}(1,2)$ and $K^{\prime}=\{(3,4)\}$ is

$$
z_{12} \geq t_{34}\left(x_{31}+x_{42}+x_{34}+x_{43}-1\right)=0.5
$$

So by introducing this inequality in the current $L P$ relaxation, we can cut off the point $(x, z)$.

This example suggests that inequalities (33) can be useful in a branch and cut framework.

\section{CONCLUSION}

We considered two formulations for the UHL that are based on flow variables. The first formulation is the multicommodity formulation. We presented two ways of projecting out the flow variables in this formulation. We determined some dicut inequalities that are dominated comparing the two projections.

Then we projected out the flow variables in the hub location formulation. The inequalities obtained from the projection of the hub location formulation include the ine- 
qualities obtained from projection applied to the multicommodity formulation.

For the hub location formulation, we characterized the extreme rays of the projection cone for the single commodity case and pointed out its relation to cuts for flows. For the multicommodity case, we identified some of the extreme rays. The projection inequalities defined by a subfamily of these rays is sufficient to have a valid formulation of UHL. We showed that some of these inequalities are facet defining while some others are dominated by other facet-defining inequalities. We also presented a family of valid inequalities that generalizes these facet-defining inequalities and that can be separated in polynomial time.

\section{Acknowledgments}

The support of France Telecom R\&D is gratefully acknowledged. The authors are grateful to two anonymous referees for their comments and suggestions.

\section{REFERENCES}

[1] P. Avella and A. Sassano, On the p-median polytope, Math Program 89 (2001), 395-411.

[2] E. Balas, "Projection and lifting in combinatorial optimiza- tion," Computational combinatorial optimization, M. Jünger and D. Naddef (Editors), Springer, Berlin, 2001, pp. 26-56.

[3] J.F. Campbell, A.T. Ernst, and M. Krishnamoorthy, "Hub location problems," Facility location: Applications and theory, Z. Drezner and H.W. Hamacher (Editors), Springer, Berlin, 2002, pp. 373-407.

[4] M. Iri, On an extension of the max-flow min-cut theorem to multicommodity flows, J Operat Res Soc Jpn 13 (1971), 129-135.

[5] M. Labbé, H. Yaman, and E. Gourdin, A branch and cut algorithm for hub location problems with single assignment, ISRO/OR Series Preprint 2003/05, Université Libre de Bruxelles, 2003. Available at http://smg.ulb.ac.be/.

[6] P. Mirchandani, Projections of the capacitated network loading problem, Eur J Operat Res 122 (2000), 534-560.

[7] K. Onaga and O. Kakusho, On the feasibility conditions of multicommodity flows in networks, IEEE Transact Circuit Theory 18 (1971), 425-429.

[8] R.L. Rardin and L.A. Wolsey, Valid inequalities and projecting the multicommodity extended formulation for uncapacitated fixed charge network flow problems, Eur J Operat Res 71 (1993), 95-109.

[9] D. Skorin-Kapov, J. Skorin-Kapov, and M. O'Kelly, Tight linear programming relaxations of uncapacitated p-hub median problem, Eur J Operat Res 94 (1996), 582-593.

[10] H. Yaman, Concentrator location in telecommunication networks, Ph.D. Thesis, Université Libre de Bruxelles, 2002. Available at http://smg.ulb.ac.be/. 